\title{
GENERATORS OF SOLAR DIFFERENTIAL ROTATION AND IMPLICATIONS OF HELIOSEISMOLOGY
}

\author{
G. RÜDIGER ${ }^{1}$ and I. TUOMINEN ${ }^{2}$ \\ ${ }^{1}$ Sternwarte Babelsberg, 1591 Potsdam, German Democratic Republic \\ ${ }^{2}$ Observatory and Astrophysics Laboratory, University of Helsinki \\ Tähtitorninmäki, SF-00130 Helsinki, Finland
}

\begin{abstract}
We interpret the helioseismological results for the solar rotation law as implying a uniform $\Omega_{0}$ in depth, if the angular velocity $\Omega(\theta)$ is expanded in series of orthogonal polynomials. One of the possibilities to ensure uniformity of $\Omega_{0}$ is to exclude any anisotropy from the generating turbulence field, so that only the rotationally originated part of the heat conductivity tensor survive as a generator of meridional flow and consequently equatorial acceleration is produced. In addition to this simplest possible turbulence model, we discuss some possible forms of the nondiffusive parts of the Reynolds stresses which may be compatible with the uniformity of $\Omega_{0}$.
\end{abstract}

\section{The solar rotation law}

The recent observational results of helioseismology have increasingly revealed the rotation law of the solar convection zone. Various inversions of the oscillation data have led to nearly the same results. Apparently the rotation law of the solar convection zone is roughly independent on depth and continues the outer well-known rotation law down to the bottom. For the lower overshooting region the reductions suggest basically rigid rotation (Dziembowski et al., 1988, Christensen-Dalsgaard and Schou, 1988). Roughly speaking the $\Omega$-contours are cylindrical in the close equatorial region, radial at higher latitudes and disk-like at the poles (cf. Fig.2 in Libbrecht, 1988). They are in particular far from the over-all cylindrical structure, $\Omega=\Omega(r \sin \theta)$, envisaged from the Taylor-Proudman theorem and from the conservation law of angular momentum under the presense of a meridional circulation with high Reynolds number. If the often used expression of the surface rotation law

$$
\Omega=A+B \cos ^{2} \theta+C \cos ^{4} \theta
$$

is transformed to the more physical series

$$
\Omega=\Omega_{0}+\Omega_{2} P_{3}^{1} / \sin \theta+\Omega_{4} P_{5}^{1} / \sin \theta+\ldots
$$

the numerical values will be

$$
\Omega_{0}=2.768, \quad \Omega_{2}=-0.087, \quad \Omega_{4}=-0.011,
$$


(in $\mu \mathrm{rad} / \mathrm{s}$ ), with a high numerical equality of the surface $\Omega_{0}$ and the interior $\Omega_{0}$. The solar rotation law seems thus to be characterized by the properties

$$
\Omega_{0}\left(r_{i}\right)=\Omega_{0}\left(r_{s}\right) \text { and } \Omega_{n}\left(r_{i}\right) \approx 0 \text { for } n>0 .
$$

Only $\Omega_{0}$ enters into the angular momentum expression

$$
J=\int \rho r^{2} \sin ^{2} \theta \Omega d v=4 \pi \int \rho r^{4} \Omega_{0}(r) d r
$$

while the remaining terms in (2) do not contribute at all. The property (4) can thus trivially be reformulated with (5) so that the angular momentum in the convection zone should be the same as if it rotates rigidly at the rate of the interior. This statement does not, however, contain more physical information than (4) already expresses (cf. Gilman et al., 1989).

\section{Differential rotation for isotropic turbulence}

The correlation tensor $\left\langle u_{i}^{\prime} u_{j}^{\prime}\right\rangle$ as well as the turbulent heat flux $\left\langle T^{\prime} u_{i}^{\prime}\right\rangle$ contain rotationally induced terms which are nonvanishing for rigid rotation. Due to their existence, rigid rotation can never be a solution of the equations. We must distinguish, however, between two different sets of parameters describing the extra terms due to the basic rotation. The first belongs to the turbulent angular momentum transport (TMT) and exists only for anisotropic turbulences. In particular, its leading term $\left(V^{(0)}\right)$ which has already been used in the Wasiutinski-Biermann-Kippenhahn formulation of the theory of differential rotation, generally produces radial gradients of the angular velocity. In opposition, the second set of parameters associated to the turbulent heat transport (THT) exists also for isotropic turbulences. They produce meridional circulation by pole-equator differences of the mean temperature. Strong radial gradients in $\Omega$ are not characteristic for their action. From this point of view the helioseismological results could indicate the action of THT-effects alone, working with isotropic turbulence. We have then a vanishing $\Lambda$-effect and the tensor of eddy conductivity is exceptionally simple. Only the rotation axis, $\boldsymbol{\Omega}$, yields a preferred direction and hence the complete tensorial structure is

$$
\chi_{i j}=P(\Omega) \delta_{i j}+Q(\Omega) \Omega_{i} \Omega_{j},
$$

so that simply

$$
\chi_{r r}=P+Q \cos ^{2} \theta, \quad \chi_{r \theta}=\chi_{\theta r}=-Q \cos \theta \sin \theta, \quad \text { and } \quad \chi_{\theta \theta}=Q \cos ^{2} \theta .
$$

Only one parameter, $Q$, describes the latitudinal dependence of the entire conductivity tensor. It can be expressed with the spectral function of $q$ of the non-rotating turbulence:

$$
Q=\frac{8}{5} \Omega^{2} \chi \int \frac{\omega k^{4}}{\left(\omega^{2}+\nu^{2} k^{4}\right)^{2}}\left[\frac{2 \omega k^{4}\left(\nu^{2}-\chi^{2}\right)}{\left(\omega^{2}+\chi^{2} k^{4}\right)^{2}} q-\frac{\omega^{2}+\nu^{2} k^{4}}{\omega^{2}+\chi^{2} k^{4}} \frac{\partial q}{\partial \omega}\right] d k d \omega
$$

$k$ and $\omega$ being wavenumber and frequency of the turbulence spectrum. The spectral function is always non-negative. The quantity $Q$ has no definite sign. It is positive for very steep 
spectral functions, $q \propto \delta(\omega)$, and it is negative for flat spectra of the "white noise"-type, $q \approx$ const and small Prandtl number.

We can numerically fix, on the other hand, the quantity $Q$ by means of the observed surface differential rotation law if the depth-dependence of $Q$ is known. With the plausible idea that already from the dimensional reasons $Q$ depends on the inverse Rossby number

$$
Q \approx\left(t_{\text {corr }} \Omega\right)^{2},
$$

we suggest $Q$ to be constant for a giant flow pattern while a relatively strong inward increase may reflect turbulence of mixing length type. We have worked thus with the depth-dependence

$$
Q \approx\left(x_{i} / x\right)^{\lambda}
$$

with free $\lambda$ and $x_{i}$ the lower fractional radius of the convection zone. As in Rüdiger (1989) and Tuominen and Rüdiger (1989) the Taylor number is $5 \times 10^{5}$, Prandtl number is 0.33 and normalized surface temperature is $T^{*}=5 \times 10^{7}$. Table 1 gives the results. Small values of $\lambda$ need positive $Q$ for the generation of the observed $\Omega_{2}$. They also give very strong basal differential rotation, much too strong poleward meridional motion, and large pole-equator temperature difference. When $\lambda=8$ there appears a reversal of the basal differential rotation, meridional flow slows down and the pole-equator temperature difference becomes reasonably small. Our models favour thus the concept of the mixing-length rather than that of the global convection. Contours of constant angular velocity are given for two models in Fig. 1 , with $\lambda=0$, and for a reasonable one with $\lambda=12$.

Table 1: Characteristic values for the isotropic turbulence models of the solar differential rotation. Angular velocity values has been here scaled so that at the surface $\Omega_{e}=1.06$ and $\Omega_{\mathrm{p}}=0.76$. The table gives for different $\lambda$ the surface values of $\Omega_{2}, Q$ at the bottom, polar and equatorial $\Omega$ at the bottom, surface meridional flow $(\mathrm{m} / \mathrm{s})$, and surface pole-equator temperature difference.

\begin{tabular}{|r|rrrrc|}
\hline$\lambda$ & $\Omega_{2} / Q_{i}$ & $Q_{i}$ & $\Omega_{p} / \Omega_{e}$ & $u_{\theta}$ & $\left(T_{e}-T_{p}\right) / T_{e}$ \\
\hline 0 & -.214 & .19 & $0.30 / 1.16$ & -63 & -.027 \\
2 & -.015 & 2.7 & $-4.16 / 1.90$ & -625 & -.18 \\
3 & .044 & -.91 & $2.12 / 0.85$ & +176 & +.045 \\
4 & .086 & -.46 & $1.40 / 0.98$ & +73 & -.011 \\
8 & .152 & -.26 & $0.96 / 1.05$ & +16 & +.0033 \\
12 & .152 & -.26 & $0.88 / 1.07$ & +4 & +.0018 \\
15 & .141 & -.28 & $0.85 / 1.07$ & -0.6 & -.0010 \\
\hline
\end{tabular}

\section{The horizontal Reynolds stress}

Let us interprete sunspot proper motions traditionally, with respect to the mean flow, i.e. with respect to the differential rotation as well as to stochastic component of the flow. As we know that the latter is reflected by the horizontal correlation $\left\langle u_{\theta}^{\prime} u_{\phi}^{\prime}\right\rangle$, first derived by means of proper motions of sunspot groups by Ward (1965). In the mean-field approach an 
isotropic turbulence field causes non-vanishing horizontal correlations via a positive eddy viscosity, defined by

$$
\left\langle u_{\theta}^{\prime} u_{\phi}^{\prime}\right\rangle=-\nu_{T} \frac{\partial \Omega}{\partial \theta} \sin \theta
$$

so that the correlation is negative in the northern hemisphere and positive in the southern. The observed signs are just opposite, so that an extra term must be added:

$$
\left\langle u_{\theta}^{\prime} u_{\phi}^{\prime}\right\rangle=-\nu_{T} \frac{\partial \Omega}{\partial \theta} \sin \theta+\nu_{T} \Omega H^{(1)} \sin ^{2} \theta \cos \theta
$$

(Rüdiger, 1977). This term is a part of the " $\Lambda$-effect" and reflects the rotational influence on the correlation tensor of anisotropic turbulences. Observations require a numerical value of about 1.6 for the quantity $H^{(1)}$. If the radial profile of $d \Omega / d \theta$ is known from helioseismology and if the radial profile of $\left\langle u_{\theta}^{\prime} u_{\phi}^{\prime}\right\rangle$ is known from statistics of the sunspot proper motions, then the radial distribution of $H^{(1)}$ can be derived containing information on the inner turbulence regime. In particular this concerns the radial distribution of the correlation time because we have similarly as in (9)

$$
H^{(1)} \approx\left(\tau_{\text {corr }} \Omega\right)^{2}
$$

According to this idea, the results from the statistics of sunspot group proper motions can be interpreted in the following way: the rotational velocity decreases with the age of groups (about 5\%) and the horizontal correlation decreases as well, being three times larger for the youngest groups than for the old recurrent ones (Tuominen and Virtanen, 1989). Let us locate the information given by the youngest groups at the botom of the convection zone and older ones correspondingly to higher layers. If we assume that the $5 \%$ outward decrease of angular velocity occurs in the lower half of the convection zone, we obtain, from the outward decreasing horizontal correlation, for the value of $\lambda$ about 6-8 in the relation (4). This outward decrease of angular velocity in the lower part of convection zone may not be in conflict with the helioseismological inversions (see Dziembovski et al., 1989).

\section{Radial $\Lambda$-effect}

$\Omega_{0}$ is also exceptional insofar as the conservation law of angular momentum allows a first integration

$$
d \Omega_{0} / d r=-6 A \Omega_{0} / 5 \rho r^{2}+\left(V^{(0)}+\frac{4}{5} V^{(1)}\right) \Omega_{0} / r
$$

with $A$ denoting the stream function of the meridional flow and $V^{(0)}$ and $V^{(1)}$ representing the $\Lambda$-effect up to the same order as $H^{(1)}$ in (12):

$$
\left\langle u_{r}^{\prime} u_{\phi}^{\prime}\right\rangle=\nu_{T}\left(-\frac{r}{\Omega} \frac{\partial \Omega}{\partial \theta}+V^{(0)}+V^{(1)} \sin ^{2} \theta\right) \sin \theta \Omega
$$

Uniform $\Omega_{0}$ is thus not only possible for vanishing $V$ 's but also if the sum $V^{(0)}+\frac{4}{5} V^{(1)}$ becomes small. Opposite signs of $V^{(0)}$ and $V^{(1)}$ are indeed characteristic for very broad classes of turbulences (Rüdiger, 1989). In Table 2 we present numerical results for such 


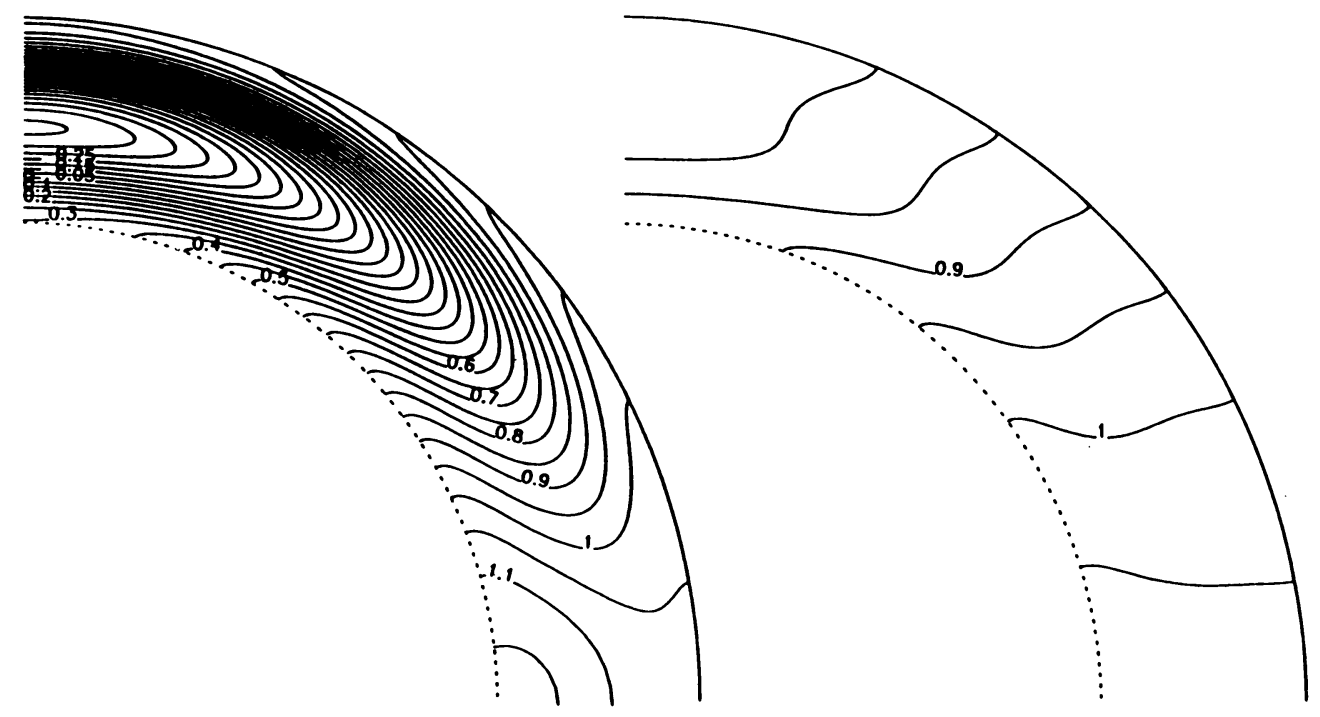

Figure 1. Angular velocity contours for the isotropic turbulence models with $\lambda=0$ (left) and $\lambda=12$ (right).

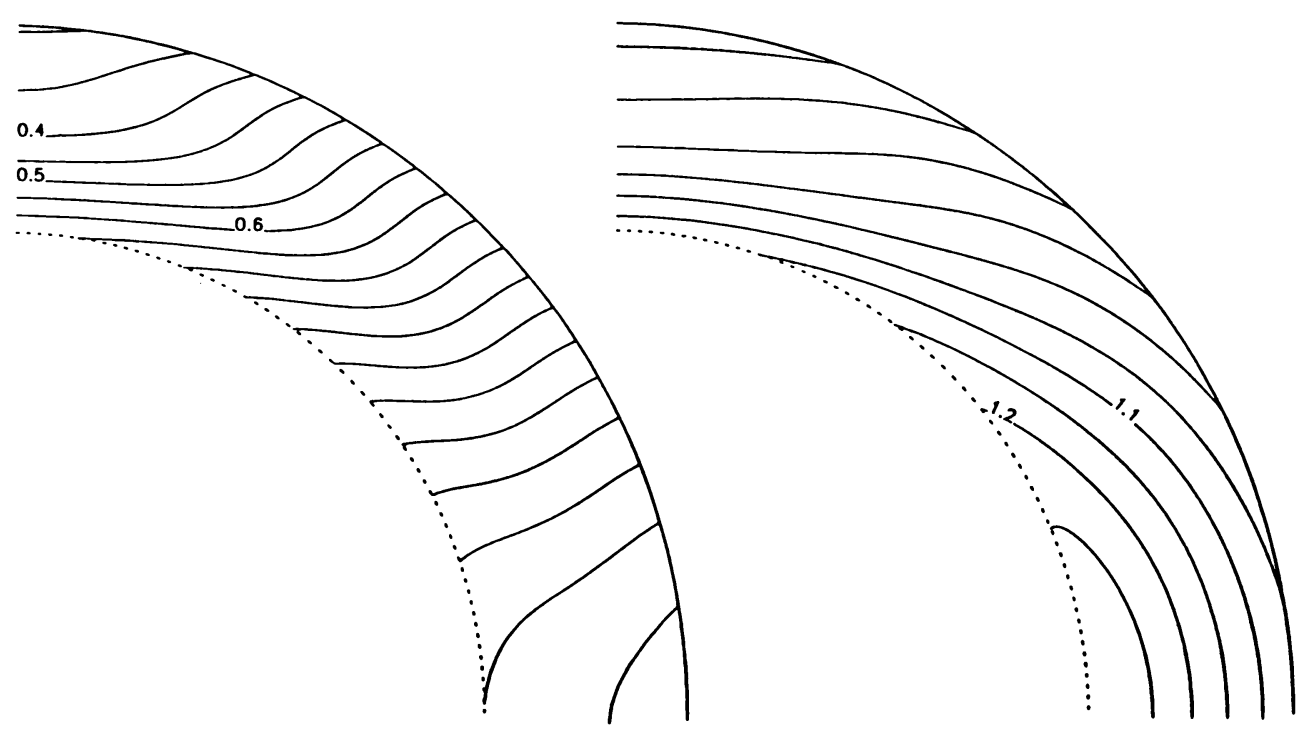

Figure 2. Angular velocity contours for the models with $\lambda=0$ (left) and $\lambda=8$ (right) for $V^{(0)}=-1$ and $V^{(1)}=5 / 4$. 
models, again with small and large $\lambda . V^{(0)}$ is taken uniform through the whole convection zone. THT-effects are neglected. The numbers clearly demonstrate the existence of some medium where the observed smooth angular velocity profile can be reproduced. A positive $V^{(0)}$ reproduces strong basal differential rotation. The circulation has correct direction and size. On the contrary, the models with a negative $V^{(0)}$ (Fig. 2) have correctly smaller differential rotation at the bottom than at the surface, although for $\lambda=0$ it is everywhere too strong. In this case, however, the $\Omega$-contours have the directions similar to observations.

The results demonstrate the complex nature of the $\Lambda$-effect. Furthermore, as we have noted earlier (Rüdiger and Tuominen, 1987; Rüdiger, 1989) with a somewhat larger Taylor number the system becomes unstable (the determinant of the linear system of equations vanishes). It is possible that in this region nonlinear effects in Reynolds stresses become important, limiting the amplitude of $\Lambda$-effect by nonlinear feedback, and influence the angular momentum balance in the convection zone.

Table 2: Characteristic numbers for the models with $\Lambda$-effect. Scaling of $\Omega$ as in Table 1 .

\begin{tabular}{|c|cccccc|}
\hline$\lambda$ & $V^{(0)}$ & $V^{(1)}$ & $H^{(1)}$ & $\Omega_{p} / \Omega_{e}$ (sur) & $\Omega_{p} / \Omega_{e}$ (bot) & $u_{\theta}$ \\
\hline 0 & 1 & $-5 / 4$ & 1 & $0.72 / 1.07$ & $0.37 / 1.16$ & -13 \\
8 & 1 & $-5 / 4$ & 1 & $0.82 / 1.04$ & $0.43 / 0.85$ & -10 \\
0 & -1 & $5 / 4$ & 1 & $0.29 / 1.18$ & $0.64 / 1.10$ & -3 \\
8 & -1 & $5 / 4$ & 1 & $0.77 / 1.06$ & $1.08 / 1.28$ & -0.2 \\
\hline
\end{tabular}

\section{References}

Christensen-Dalsgaard, J., Schou, J.: 1988, Proc. Symp. Seismology of the Sun and Sun-like Stars, ESA SP-286, 149.

Dziembowski, W.A., Goode, P.R., Libbrecht K.G.: 1989, Astrophys. J. 337, L53.

Gilman, P.A., Morrow, C.A., DeLuca, E.E.: 1989, Astrophys. J. 338, 528.

Libbrecht, K.G.: 1988, Proc. Symp. Seismology of the Sun and Sun-like Stars, ESA SP-286, 131.

Rüdiger, G.: 1977, Astron. Nachr. 298, 245.

Rüdiger, G.: 1989, Differential Rotation and Stellar Convection: Sun and Solar-type stars, Gordon and Breach, New York.

Rüdiger, G., Tuominen, I.: 1987, in The Internal Solar Angular Velocity, eds. B.R. Durney and S. Sofia, D. Reidel, Dordrecht, p. 361.

Tuominen, I., Rüdiger, G.: 1989, Astron. Astrophys. 217, 217.

Tuominen, I., Virtanen, H.: 1989, to be submitted to Solar Phys.

Ward, F.: 1965, Astrophys. J. 141, 534. 